\title{
p38 pathway targets SWI-SNF chromatin-remodeling complex to muscle-specific loci
}

\author{
Cristiano Simone ${ }^{1,2,4}$, Sonia Vanina Forcales ${ }^{1,4}$, David A Hill ${ }^{3}$, Anthony N Imbalzano ${ }^{3}$, Lucia Latella ${ }^{1}$ \& \\ Pier Lorenzo Puri ${ }^{1,2}$
}

\begin{abstract}
During skeletal myogenesis, genomic reprogramming toward terminal differentiation is achieved by recruiting chromatinmodifying enzymes to muscle-specific loci ${ }^{1,2}$. The relative contribution of extracellular signaling cascades in targeting these enzymes to individual genes is unknown. Here we show that the differentiation-activated p38 pathway ${ }^{3-5}$ targets the SWI-SNF chromatin-remodeling complex to myogenic loci. Upon differentiation, p38 kinases were recruited to the chromatin of muscle-regulatory elements. Blockade of $p 38 \alpha / \beta$ repressed the transcription of muscle genes by preventing recruitment of the SWI-SNF complex at these elements without affecting chromatin binding of muscle-regulatory factors and acetyltransferases. The SWI-SNF subunit BAF60 could be phosphorylated by $\mathrm{p} 38 \alpha-\beta$ in vitro, and forced activation of $\mathrm{p} 38 \alpha / \beta$ in myoblasts by expression of a constitutively active MKK6 (refs. 5-7) promoted unscheduled SWI-SNF recruitment to the myogenin promoter. Conversely, inactivation of SWI-SNF enzymatic subunits abrogated MKK6-dependent induction of muscle gene expression. These results identify an unexpected function of differentiation-activated p38 in converting external cues into chromatin modifications at discrete loci, by selectively targeting SWI-SNF to muscle-regulatory elements.
\end{abstract}

Pharmacological blockade of $\mathrm{p} 38 \alpha / \beta$ kinases by SB203580 inhibits the myogenic program ${ }^{3-5}$ by repressing the transcription of early (myogenin; $\mathrm{Myog}$ ) and late (muscle-creatine kinase; $\mathrm{Ckm}$ ) muscle genes in myoblasts induced to differentiate (Fig. 1a). We monitored by chromatin immunoprecipitation (ChIP) the effect of p38 inhibition on the assembly of the myogenic transcriptosome and on chromatin modifications at the $M y o g$ promoter and the $C \mathrm{~km}$ enhancer during myoblast differentiation. The muscle-regulatory factors (MRFs) MyoD and MEF2C bound the Myog promoter within the first hours of incubation in differentiation medium (Supplementary Fig. 1 online) and throughout the differentiation process, regardless of the presence of SB203580 (Fig. 1b). At the Ckm enhancer, MRF binding was delayed (data not shown) and not influenced by SB203580 (Fig. 1b).p38 $\alpha$ was selectively recruited to chromatin occupied by MRFs during muscle differentiation (Fig. 1b), as MRFs and p38 were not detected at these sequences in undifferentiated myoblasts (Fig. 1b) or in fibroblasts cultured in differentiation medium (data not shown). MRFs and p38 were also not found on the coding regions of $\mathrm{Ckm}$ (Supplementary Fig. 2 online) or on the Igh enhancer (Fig. 1b), which contains E-boxes occupied by E-proteins only in $\mathrm{B}$ cells ${ }^{8}$. An interaction between Mef2A-C and p38 was suggested by previous studies ${ }^{4,5,9,10}$, and MyoD could bind p38 in vitro (Supplementary Fig. 2 online), suggesting that MRFs might mediate 338 recruitment to muscle-regulatory elements.

We next investigated the effect of p38 inhibition on chromatin modifications produced by MRF-recruited enzymes, including $\mathrm{p} 38$, histone acetyltransferases (p300, PCAF) $)^{1,2,11,12}$ and the chromatin-remodeling SWI-SNF complex ${ }^{13}$. In undifferentiated myoblasts, histones $\mathrm{H} 4$ and $\mathrm{H} 3$ within the regulatory elements of $\mathrm{Myog}$ and $\mathrm{Ckm}$ were hypoacetylated and hypophosphorylated (Fig. 1b). We detected hyperacetylation of histones and phosphoacetylation of histone $\mathrm{H} 3$ at the Myog promoter and the $\mathrm{Ckm}$ enhancer after incubation in differentiation medium in cells treated with SB203580 and in untreated cells, which at times correlated with MRF binding (Fig. 1b and Supplementary Fig. 1 online). In contrast, treatment with SB203580 eliminated the increased phosphoacetylation of histone $\mathrm{H} 3$ at the Jun promoter caused by TNF $\alpha$ in myoblasts (Supplementary Fig. 3 online). These results suggest that p38 is involved in phosphoacetylation of histone $\mathrm{H} 3$ at promoters activated by inflammatory cytokines ${ }^{14}$, but not muscle-specific promoters.

Histone hyperacetylation reflects the activity of acetyltransferases recruited by MRFs. Previous reports showed that p300-CBP and PCAF directly acetylate MyoD ${ }^{15,16}$. We evaluated the acetylation status of MyoD at the regulatory elements of $M y o g$ and $C k m$ by using antibodies to acetylated $\mathrm{MyoD}^{17}$. Acetylated $\mathrm{MyoD}$ was detectable at the Myog promoter after a few hours in differentiation medium (Fig. 1b and Supplementary Fig. 1 online) and at the $C \mathrm{~km}$ enhancer after 18-24 h in differentiation medium (Fig. 1b). The distinct kinetics of chromatin binding of acetylated MyoD at these

${ }^{1}$ Laboratory of Gene Expression, Dulbecco Telethon Institute at Fondazione A. Cesalpino, Institute of Cell Biology and Tissue Engineering, San Raffaele Biomedical Science Park of Rome, Rome 00128, Italy. ${ }^{2}$ The Salk Institute for Biological Studies, Peptide Biology Laboratory, La Jolla, California 92093 , USA. ${ }^{3}$ University of Massachusetts Medical School, Department of Cell Biology, Worcester, Massachusetts 01655, USA. ${ }^{4}$ These authors equally contributed to this work. Correspondence should be addressed to P.L.P. (plpuri@dti.telethon.it).

Published online 20 June 2004; doi:10.1038/ng1378 
regulatory regions correlated with the presence of PCAF (Fig. 2a), which acetylates MyoD at residues recognized by this antibody ${ }^{18}$. Exposure to SB203580 affected neither the amount of chromatinbound acetylated MyoD (Fig. 1b) nor the acetyltransferase activity copurified by antibodies to MyoD from nuclear extracts of myoblasts induced to differentiate (Fig. 1c). As local hyperacetylation is not influenced by $\mathrm{p} 38 \alpha / \beta$, we considered that a further step in transcription activation, chromatin remodeling, could be the functional target of the p38 pathway. MyoD-associated chromatin remodeling activity has previously been described ${ }^{19}$. Changes in chromatin structure at the endogenous $\mathrm{Myog}$ and $\mathrm{Ckm}$ loci underlies the transcription of these genes during differentiation and can be monitored by restriction endonuclease access to specific sites $^{13,19}$. We observed two peaks of endonuclease accessibility at the BanI site of the Myog promoter, after $6 \mathrm{~h}$ and $24 \mathrm{~h}$ in differentiation medium (Fig. 1d), whereas the $C \mathrm{~km}$ enhancer was accessible to endonucleases only after $18 \mathrm{~h}$ in differentiation medium (Fig. 1e). Chromatin remodeling at both loci was precluded by SB203580 (Fig. 1d,e), suggesting that $\mathrm{p} 38 \alpha / \beta$ stimulates musclegene transcription by promoting recruitment or activation of the SWI-SNF chromatin-remodeling complex.

We therefore monitored the effect of SB203580 on the recruitment of acetyltransferases and the enzymatic subunits of the SWI-SNF complex (BRG1 and BRM) at the Myog promoter and Ckm enhancer in differentiating myoblasts. p300 and PCAF were found on the Myog promoter a few hours after incubation in differentiation medium (Fig. 2a), and p300 was detected at the Ckm enhancer during the first $18 \mathrm{~h}$ in differentiation medium (Fig. 2a). PCAF bound the $\mathrm{Ckm}$ enhancer at later stages of differentiation instead (Fig. 2a), coincident with the late binding of acetylated MyoD and the late onset of $\mathrm{Ckm}$ expression (Fig. 1a,b). BRG1 was reproducibly detected at $M y o g$ and $\mathrm{Ckm}$ regulatory elements after incubation in differentiation medium using three different antibodies to BRG1 (Fig. 2a and Supplementary Fig. 4 online). We also detected BRM at the Myog promoter but found a much smaller amount at the $\mathrm{Ckm}$ enhancer (Fig. 2a), suggesting that distinct complexes, with potential redundant functions, might be recruited at muscle-regulatory sequences, possibly by different $\mathrm{MRFs}^{18}$.

Inhibition of $\mathrm{p} 38 \alpha / \beta$ did not affect the recruitment of p300 and PCAF (Fig. 2a) but prevented the engagement of BRG1 and BRM at the Myog promoter and the Ckm enhancer (Fig. 2a), supporting the conclusion that chromatin remodeling is selectively influenced by p38 $\alpha / \beta$. Recruitment of SWI-SNF complex has been linked to the engagement of the hyperphosphorylated, active fraction of RNA polymerase II, a crucial step in transcription initiation ${ }^{20}$. Consistent with this notion, the inhibition of BRG1-BRM recruitment by SB203580 correlated with reduced levels of RNA polymerase II phosphorylated at Ser5 at the Myog promoter and Ckm enhancer (Fig. 2a). In contrast, RNA polymerase II phosphorylated at Ser2 was detected only in the coding region of transcribed $\mathrm{Ckm}$ (Supplementary Fig. 2 online).

Immunoprecipitation of nuclear extracts with antibodies to MyoD uncovered an association between endogenous MyoD, p300, PCAF and BRG1 in differentiating myoblasts (Fig. 2b), but only the MyoDBRG1 interaction was disrupted by SB203580 (Fig. 2b). SB203580 did not decrease the cellular levels of BRG1 or other SWI-SNF components (Fig. 2c). MyoD-Mef2 interactions have been previously described $^{21}$. As antibodies to MyoD could coprecipitate Mef2 and vice versa, and antibodies to Mef2 also coprecipitated BRG1 (data not shown), the SWI-SNF complex might interact with MyoD, Mef2 or both.
Figure 1 Blockade of $\mathrm{p} 38$ by SB203580 inhibits the expression of muscle genes by selectively preventing chromatin remodeling at their regulatory regions. (a) Expression of $\mathrm{Myog}, \mathrm{Ckm}$ and Gapd RNA was monitored by RT-PCR with RNA from $\mathrm{C} 2 \mathrm{C} 12$ undifferentiated myoblasts cultured in growth medium (GM) or induced to differentiate by incubation in differentiation medium (DM) for the indicated times, exposed (+) or not exposed to SB203580 (SB). (b) ChIP analysis of E-box-containing regions in the Myog promoter and the $\mathrm{Ckm}$ and Igh enhancers was done in undifferentiated (GM) or differentiated (DM) $\mathrm{C} 2 \mathrm{C} 12$ myoblasts, in the presence (+) or absence of SB203580 (SB), with the indicated antibodies. (c) MyoD-associated acetyltransferase activity was monitored after MyoD

immunoprecipitation from nuclear extracts of undifferentiated (GM) or differentiated (DM) C2C12 myoblasts, in the absence (NT) or presence (SB) of SB203580 or the MEK1 inhibitor PD. Levels of immunoprecipitated MyoD were detected by western blot (WB, top panel), and the associated acetyltransferase activity was evaluated by $\mathrm{C}^{14}$ incorporation (middle panel) of free histones (stained with Coomassie, bottom panel) after in vitro acetylation assay. Relative activation quantifies the histone incorporation of $\mathrm{C}^{14}$ acetylated coenzyme $\mathrm{A}$ relative to the $\mathrm{C}^{14}$ incorporation detected in untreated undifferentiated myoblasts (lane 1 ) and is normalized to the levels of immunoprecipitated MyoD. (d,e) Restriction endonuclease accessibility was done on nuclei isolated from C2C12 myoblasts either undifferentiated (GM) or differentiated by incubation in differentiation medium (DM) for the indicated times, with or without SB203580 (SB). Nuclei were digested with Banl and then subjected to PCR to amplify a 197-bp fragment from -106 to +91 relative to the transcription start site (TSS) for the Myog promoter (d) or a fragment from $-1,256$ to $-1,122$ relative to the transcription start site (TSS) for the $\mathrm{Ckm}$ enhancer (e). As a control, a p21 promoter fragment devoid of Banl sites was used. Absence or reduction of PCR signal indicates Banl accessibility and reflects chromatin remodeling. 
Figure 2 Blockade of $\mathrm{p} 38$ by SB203580 selectively prevents SWI-SNF recruitment at regulatory regions of muscle genes. (a) ChIP analysis of E-box-containing regions in the Myog promoter and the $\mathrm{Ckm}$ and $\mathrm{lgh}$ enhancers was done in $\mathrm{C} 2 \mathrm{C} 12$ myoblasts undifferentiated (GM) or differentiated (DM) for the indicated times, in the presence (+) or absence of SB203580 (SB), by using the described antibodies. Densitometric analysis showed that binding of BRG1 to chromatin was progressively reduced in cells treated with SB203580 at the Myog promoter (by $70 \%$ at $18 \mathrm{~h}$ and $90 \%$ at $36 \mathrm{~h}$ ) and at the $\mathrm{Ckm}$ enhancer (by $55 \%$ at $18 \mathrm{~h}$ and $90 \%$ at $36 \mathrm{~h}$ ).

(b) Immunoprecipitation of MyoD was done from nuclear extracts of undifferentiated (GM) or differentiated (DM) C2C12 myoblasts in the presence (+) or absence of SB203580 (SB) Levels of precipitated MyoD and coprecipitated p300, PCAF and BRG1 were detected by western blotting. (c) Expression levels of BRG1, BRM, BAF60c, BAF47 and GAPDH RNA were monitored by RT-PCR (left panel) and protein levels of BRG1, MyoD and tubulin were monitored by western blotting (right panel) from $\mathrm{C} 2 \mathrm{C} 12$

myoblasts cultured in growth medium (GM) or in differentiation medium (DM), with (+) or without SB203580 (SB). (d) A kinase assay was carried out by incubating the mammalian SWI-SNF complex with P32-ATP in the presence or absence of recombinant p38 kinases $\alpha / \beta$ and SB203580 (SB). A fraction of each reaction was used for western blotting (WB) with antibodies to FLAG-tagged Ini-BAF47 to normalize the levels of the complex used for the reaction. A kinase reaction (KA) with recombinant $\mathrm{p} 38$ kinases $\alpha / \beta$ and their known substrate myelin basic protein (MBP) was used as positive control.
To begin elucidating the mechanism that mediates p38-dependent recruitment of SWI-SNF complex to myogenic loci, we carried out an in vitro kinase assay with purified mammalian SWI-SNF complex incubated with recombinant $\mathrm{p} 38 \alpha / \beta$ kinases. Incorporation of $\mathrm{P}^{32}$ by some SWI-SNF subunits was apparent in the absence of p38 kinases, but phosphorylation of BAF60 was induced only after incubation with p38 kinases and was eliminated by the inclusion of SB203580 (Fig. 2d). Although the functional relevance of this phosphorylation has yet to be established, it is notable that BAF60a-BAF60c isoforms have been implicated as the surface for interactions between SWI-SNF and transcriptional activators 22,23 .

We next used a C2C12 subclone stably expressing the p38 activator MKK6EE under the negative control of a tetracycline-responsive element to evaluate whether the ability of MKK6EE to override the antidifferentiation cues in myoblast ${ }^{5,6}$ relies on forced recruitment of SWI-SNF at myogenic loci. Expression of MKK6EE by
Figure 3 Forced activation of p38 in myoblasts by ectopic expression of MKK6EE induces myogenin expression by promoting BRG1 recruitment on the Myog promoter. (a) Immunoprecipitation of MyoD was done from nuclear extracts of $\mathrm{C} 2$ MKK6EE myoblasts cultured in growth medium (GM), with (+) or without (-) tetracycline (Tet) and SB203580 (SB). Levels of precipitated MyoD and coprecipitated PCAF, p300 and BRG1 were detected by western blotting. (b) ChIP analysis of E-box-containing regions in the Myog promoter was done in C2-MKK6EE myoblasts cultured in growth medium (GM), with (+) or without (-) tetracycline (Tet) and SB203580 (SB, left panel) or in the parental $\mathrm{C} 2 \mathrm{C} 12$ cell line, exposed to TNF $\alpha\left(10 \mathrm{ng} \mathrm{ml}^{-1}\right)$ in the presence or absence of SB203580 (right panel), using the indicated antibodies. (c-e) $\mathrm{C} 2 \mathrm{C} 12$ myoblasts were either infected with adenovirus expressing MKK6EE (+) or mock-infected (-) and then incubated in growth medium (GM). DM, differentiation medium. (c) Western-blot analysis was done from whole-cell extracts of MKK6EE- or mock-infected C2C12 cells to determine levels of MKK6EE (HAtagged), total and phosphorylated p38, myogenin and tubulin. (d) RT-PCR analysis of myogenin RNA from MKK6EE- or mock-infected C2C12 cells, in the presence (+) or absence of SB203580 (SB). (e) ChIP analysis of E-box-containing regions in the Myog promoter was done in MKK6EE- or mock-infected C2C12 cells, with (+) or without SB203580 (SB). a

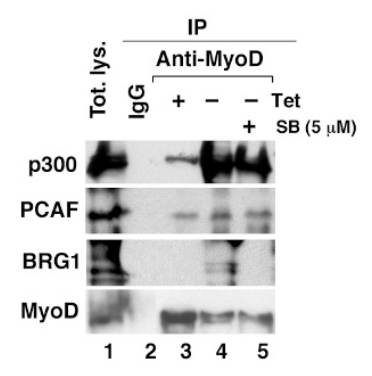

b
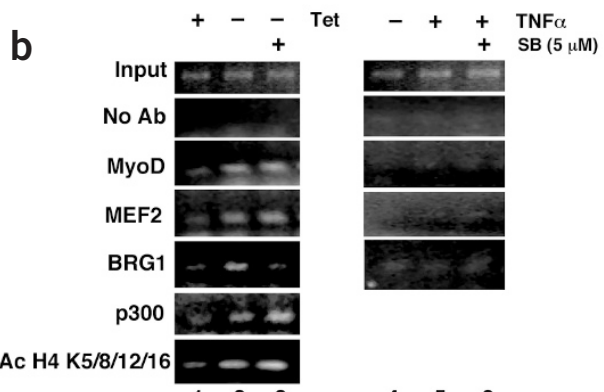

456

e
C

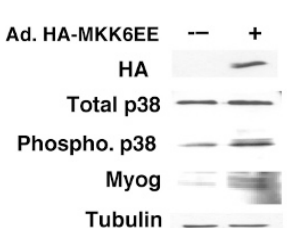

d

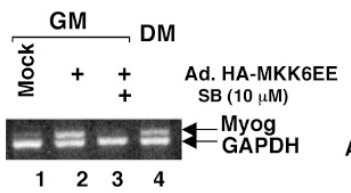


Figure 4 Inhibition or absence of SWI-SNF enzymatic activity abrogates MKK6EE-induced activation of muscle gene transcription and skeletal myogenesis. (a) C2-MKK6EE myoblasts cultured in growth medium in the presence of tetracycline were microinjected with a mixture of GFP cDNA and antibodies against BRG1, BRM, cJun and IgG. After microinjection, cells were incubated in growth medium without tetracycline for $36 \mathrm{~h}$ and fixed for immunofluorescence studies. Productively injected cells were visualized by GFP expression (green) and differentiation was visualized by staining with antibody to $\mathrm{MHC}$ (red). Nuclei were counterstained with DAPI. The differentiation of injected cells was visualized by merging the red and green images, resulting in yellow staining. The number of cells with yellow staining (MHC-GFP double positive) and the number of cells with green staining (at least 50 in each experiment) were counted in two distinct experiments to measure the effect of antibody injection on myoblast differentiation. (b) SW13 polyclones with a chromatin-integrated Gal4-luc were transfected with wild-type Gal4-MyoD (left) or Gal4-MyoD-N (right; amino acids 1-53 of MyoD) with or without MKK6EE, wild-type (WT) BRG1, enzymatic deficient BRG1 K798R (K/R) and wild-type (WT) $\mathrm{BRM}$, and then cultured for $48 \mathrm{~h}$ in growth medium in the presence or absence of SB203580 (SB). Cells were then collected and luciferase activity was measured. a

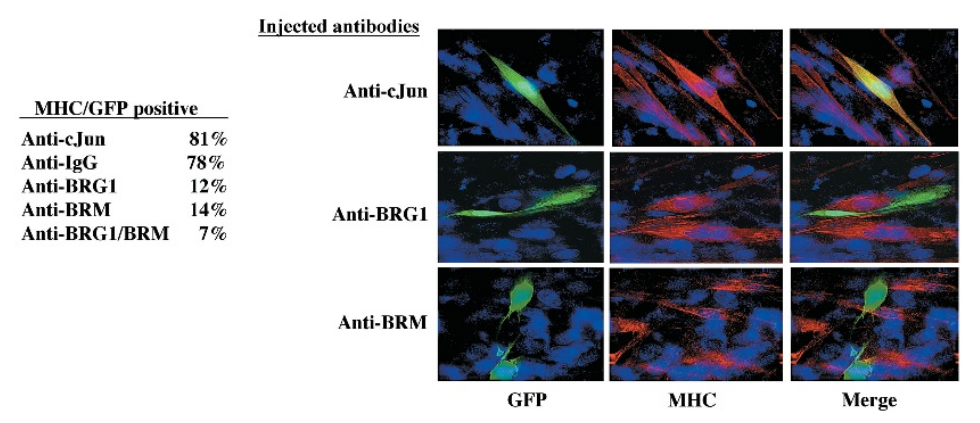

b

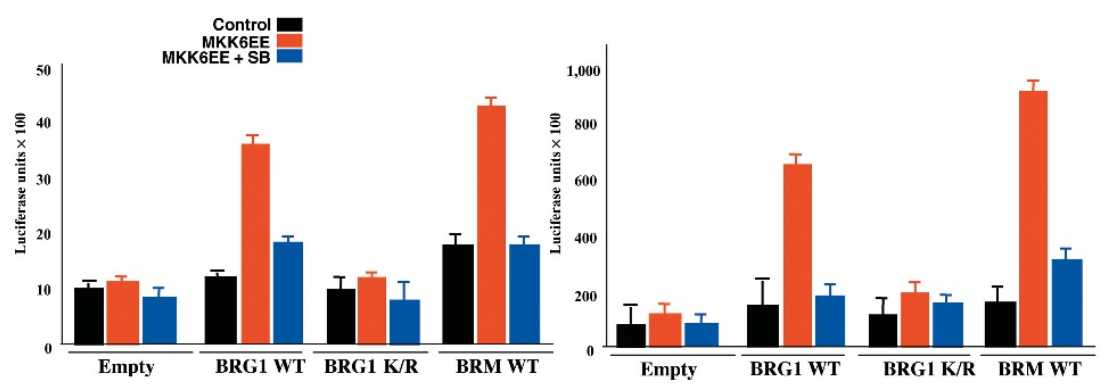

tetracycline removal in myoblasts led to the formation of a complex containing MyoD, p300, PCAF and BRG1 (Fig. 3a); SB203580 prevented BRG1 association, but not MyoD, p300 or PCAF interactions (Fig. 3a). MyoD, MEF2C, p300 and BRG1 occupied the Myog promoter after expression of MKK6EE, leading to histone hyperacetylation (Fig. 3b). Inhibition of $\mathrm{p} 38 \alpha / \beta$ selectively prevented BRG1 recruitment (Fig. $3 \mathbf{b}$ ). These results indicate that MKK6EE can promote the assembly of the myogenic transcriptosome on the Myog promoter. Only SWI-SNF recruitment seemed to be directed by $\mathrm{p} 38 \alpha / \beta$, as it was abrogated by SB203580. This suggests that other MKK6-activated, SB203580-resistant kinases might promote the recruitment of MRFs and acetyltransferases. Activation of p38 also occurs in response to inflammatory cytokines ${ }^{9}$, but p38 activation by TNF $\alpha$ does not promote any step of the transcriptosome assembly on the Myog promoter, including MRFs and BRG1 recruitment (Fig. 3b).

Acute expression of hemagglutinin (HA)-tagged MKK6EE in myoblasts was also achieved by adenoviral infection. Expression of HA-MKK6EE was detected only in infected myoblasts and correlated with p38 activation and Myog expression (Fig. 3c). SB203580 inhibited MKK6EE-induced Myog transcription (Fig. 3d). BRG1, MyoD and hyperacetylated histones were detected at the Myog promoter in myoblasts infected with MKK6EE but not in mock-infected myoblasts (Fig. 3e). Only BRG1 recruitment was inhibited by blockade of p38 $\alpha / \beta$ (Fig. 3e). To establish a causal relationship between MKK6EEp38 and SWI-SNF-dependent chromatin remodeling at the muscle loci, we inactivated the enzymatic subunits of the SWI-SNF complex by microinjection of neutralizing antibodies in $\mathrm{C} 2 \mathrm{C} 12$ cells stably expressing MKK6EE under the control of a tetracycline-responsive element. Antibodies against BRG1 or against BRM prevented MKK6EE-induced expression of muscle genes, such as myosin heavy chain (MHC; Fig. 4a).
We further investigated the requirement for SWI-SNF-dependent chromatin remodeling in MKK6EE-p38-dependent activation of MyoD-mediated transcription by monitoring the ability of Gal4-MyoD to activate a chromatin-integrated Gal4-responsive reporter (Gal4-luc) in BRG1-BRM-deficient SW13 cells ${ }^{24}$. Gal4-MyoD alone was unable to activate transcription, and MKK6EE did not augment Gal4-luc activity in these cells (Fig. 4b). Reintroduction of BRG1 and BRM, but not of the enzymatically defective BRG1 point mutant K798R (ref. 25), restored the ability of MKK6EE to activate Gal4-luc (Fig. 4b), further showing the importance of the catalytic SWI-SNF subunits in enabling MKK6EE to promote MyoD-dependent transcription. SB203580 abrogated MKK6EE-dependent activation of Gal4-MyoD in the presence of BRG1 or BRM (Fig. 4b), indicating that $\mathrm{p} 38 \alpha / \beta$ is essential in promoting SWISNF-mediated activation of MyoD-dependent transcription. We made similar observations when we fused the $\mathrm{N}$-terminal acidic transactivation domain of MyoD to Gal4 (Gal4-MyoD-N). As this mutant does not bind Mef2 (ref. 21) and induces transcription autonomously, activation of MyoD-dependent transcription by the MKK6EE-p38-SWI-SNF pathway does not seem to rely on p38-mediated phosphorylation of Mef2A-Mef2C (refs. 4,5,9,10).

Notably, Gal4-MyoD-N does not contain any of the domains previously reported to mediate activation of genes in repressive chromatin ${ }^{19}$. But SWI-SNF can be targeted to promoters by interactions with the acidic transactivation domain ${ }^{26}$ or other regions ${ }^{27}$ of sequence-specific transcriptional activators, suggesting that interactions of SWI-SNF with transcription factors are multifaceted. Local histone hyperacetylation was proposed to stabilize SWI-SNF binding to the chromatin in the absence of transcription factors ${ }^{28}$. The uncoupling of histone hyperacetylation and chromatin remodeling on muscle-regulatory regions, after blockade of $\mathrm{p} 38$, indicates that additional events, such as the activation of signaling cascades, are required to target the SWI-SNF complex to particular loci in response to specific cues. 
Our results extend the function of p38 kinases beyond their ability to activate gene expression by direct phosphorylation of transcription factors and establish a link between the differentiation-activated p38 pathway and the recruitment of SWI-SNF complexes to transcriptionally active loci during skeletal myogenesis.

\section{METHODS}

Cell cultures, transfections and luciferase assay. We cultured $\mathrm{C} 2 \mathrm{C} 12$ myoblasts and SW13 cells in Dulbecco's modified Eagle medium supplemented with $15 \%$ fetal bovine serum (growth medium). Differentiation was induced by incubation in Dulbecco's modified Eagle medium supplemented with 2\% horse serum and insulin-transferrin (differentiation medium). We generated SW13 polyclones containing integrated Gal4-luc by transfecting cells with the Gal4luc reporter and pcDNA3-neo ${ }^{\mathrm{r}}$ and selecting G418-resistant clones. We combined antibiotic-resistant colonies to yield a polyclonal population of reporter cells. C2-MKK6EE cells are described in ref. 5.

We purchased SB203580 (final concentration $5 \mu \mathrm{M}$ ), PD98059 (final concentration $25 \mu \mathrm{M}$ ) and TNF $\alpha$ (final concentration $10 \mathrm{ng} \mathrm{ml}^{-1}$ ) from Calbiochem. We added SB203580 and PD98059 to cell cultures when placed in differentiation medium and added fresh compounds every $18 \mathrm{~h}$. Transfections were done with the Lipofectamine 2000 reagent. We determined luciferase activity with a Promega Luciferase kit and normalized it either to activity for $\beta$ galactosidase or using the Dual-Luciferase reporter assay system (Promega).

Adenoviral infections. We excised a cDNA encoding MKK6EE from pcDNA3.1-MKK6EE-HA with HindIII and $\mathrm{XbaI}$ and ligated it into pAdShuttle-CMV digested with HindIII and XbaI to create pShuttle-MKK6EE. We created replication-deficient adenovirus expressing MKK6EE from the CMV promoter by homologous recombination of pShuttle-MKK6EE with pAdEasy in Escherichia coli BJ5183, generating pAdMKK6EE. The virus was multiplied by transfection of 293 packaging cells. We infected C2C12 cells with pAdMKK6EE for $2 \mathrm{~h}$ in serum-free medium and then placed them in growth medium for an additional $18 \mathrm{~h}$, with or without SB203580 (10 $\mu \mathrm{M})$.

RT-PCR. We extracted RNA from $\mathrm{C} 2 \mathrm{C} 12$ cells with Trizol Reagent (Invitrogen). We carried out RT-PCR using SuperScript One-Step RT-PCR with platinum Taq (Invitrogen). We mixed $400 \mathrm{ng}$ of total RNA with $10 \mu \mathrm{M}$ of specific primers. The RT-PCR reaction was $50^{\circ} \mathrm{C}$ for $40 \mathrm{~min}$, followed by $94^{\circ} \mathrm{C}$ for $2 \mathrm{~min} ; 25-30$ cycles of $94^{\circ} \mathrm{C}$ for $15 \mathrm{~s}, 50-55^{\circ} \mathrm{C}$ for $30 \mathrm{~s}$ and $72{ }^{\circ} \mathrm{C}$ for $40 \mathrm{~s}$; and a final step of $72{ }^{\circ} \mathrm{C}$ for $5 \mathrm{~min}$. Primer sequences are available on request.

Kinase assay. We incubated purified recombinant $p 38 \alpha / \beta$ with FLAG-tagged Inil SWI-SNF complex purified from Hela cells ${ }^{29}$, in the presence or absence of ATP and SB203580. We carried out the reaction as described previously ${ }^{5,6}$ and detected phosphorylation by $\mathrm{P}^{32}$ autoradiography.

Immunoprecipitation studies, acetyltransferase assay and western blotting We precleared nuclear cell extracts with an $A+G$ sepharose mix for 30 min at $4{ }^{\circ} \mathrm{C}$ and immunoprecipitated them with agarose-conjugated antibodies to MyoD (M-318 Ac, Santa Cruz and RGB7; ref. 12). We washed the immunoprecipitates extensively with lysis buffer, resuspended them in Laemmli buffer, separated them on polyacrylamide gels and transferred them to nitrocellulose membranes. We visualized precipitated proteins by ECL (NEN Life Sciences) with antibodies to p300 (N15, Santa Cruz), PCAF (H-369, Santa Cruz), MyoD (M-318, Santa Cruz) and BRG1 (monoclonal G-7, Santa Cruz). We carried out an in vitro acetylation assay after immunoprecipitation with antibodies to MyoD as described ${ }^{15}$. We carried out western blotting of whole-cell lysates using antibodies against HA (F7 from Santa Cruz), total p38 and phosphorylated p38 (Cell Signaling), and myogenin (FD5 hybridoma).

ChIP. We carried out a ChIP assay according to instructions from Upstate Biotechnology. We used antibodies to MyoD (M-318 Ac, Santa Cruz), Mef2c, p38 $\alpha$ (refs. 5,6), acetylated H4, acetylated $\mathrm{H} 3$ and $\mathrm{H} 3$ phosphorylated at Ser10 and acetylated at Lys14 (Upstate Biotechnology), acetylated MyoD (ref. 17), p300 (N-15 Ac, Santa Cruz), PCAF (H-369, Santa Cruz), BRG1 (ref. 13), BRG1 (polyclonal N-15 and H-88, Santa Cruz), BRM (polyclonal N19 and C20, Santa Cruz) and RNA polymerase II phosphorylated at Ser5 (H14, Covance). We carried out
PCR analysis of 'input' DNA of different samples with specific primers. PCR conditions were $95^{\circ} \mathrm{C}$ for $2 \mathrm{~min}$, followed by $25-30$ cycles at $95^{\circ} \mathrm{C}$ for $30 \mathrm{~s}, 55-60^{\circ} \mathrm{C}$ for $30 \mathrm{~s}$ and $72^{\circ} \mathrm{C}$ for $30 \mathrm{~s}$. Primer sequences are available on request.

Endonuclease assay. We extracted genomic DNA from C2C12 cells by resuspending cell pellets in RSB buffer (10 mM Tris- $\mathrm{HCl}(\mathrm{pH} 7.4), 10 \mathrm{mM} \mathrm{NaCl}$, $5 \mathrm{mM} \mathrm{MgCl} 2,0.1 \%$ Nonidet P-40 and $5 \mathrm{mM}$ butyrate, supplemented with protease and phosphatase inhibitors) and incubating for $20 \mathrm{~min}$ on ice. We homogenized nuclei with a 27 -gauge syringe, centrifuged them at 2,000 r.p.m. for 5 min at $4{ }^{\circ} \mathrm{C}$, washed them in RSB buffer, centrifuged them again at 2,000 r.p.m. for $5 \mathrm{~min}$ and resuspended them in $50 \mu \mathrm{l}$ of RSB buffer. We digested one to five nuclei in $400 \mu \mathrm{l}$ of $1 \times$ NEB4 (New England Biolabs) with $100 \mathrm{U}$ of BanI enzyme (for $1 \mathrm{~h}$ at $37^{\circ} \mathrm{C}$ ). Reactions were stopped with proteinase $\mathrm{K}$ and $2 \%$ $\mathrm{SDS}$ for $2 \mathrm{~h}$ at $45^{\circ} \mathrm{C}$. We extracted DNA with phenol-chloroform twice, precipitated it in ethanol and resuspended it in water. For PCR, we resuspended an aliquot of each DNA sample in PCR SuperMix (Invitrogen) with specific primers. PCR conditions were $94^{\circ} \mathrm{C}$ for $2 \mathrm{~min}$, followed by 25-30 cycles of $94{ }^{\circ} \mathrm{C}, 58^{\circ} \mathrm{C}$ for $30 \mathrm{~s}, 72{ }^{\circ} \mathrm{C}$ for $30 \mathrm{~s}$ and $72 \mathrm{C}$ for $7 \mathrm{~min}$. Primer sequences are available on request.

Microinjection studies and immunofluorescence. We microinjected C2MKK6EE cells with a mixture of GFP cDNA (final concentration of $0.3 \mu \mathrm{g} \mu \mathrm{l}^{-1}$ ) and antibodies against BRG1 (H88 rabbit, N15 goat or G7 mouse, each at a final concentration of $125 \mu \mathrm{g} \mathrm{ml}^{-1}$ ) or BRM (N19 goat or C20 goat, each at a final concentration of $\left.125 \mu \mathrm{g} \mathrm{m}^{-1}\right)$ alone or in combination, and cJun (H79 rabbit, final concentration of $125 \mu \mathrm{g} \mathrm{ml}^{-1}$ ) or IgG as controls. We placed cells in growth medium without tetracycline $4 \mathrm{~h}$ after microinjection. After $36 \mathrm{~h}$, cells were fixed and processed for immunofluorescence studies, using either mouse monoclonal or rabbit polyclonal antibodies to MHC, depending on the species of the injected antibodies, to avoid cross-reaction.

Note: Supplementary information is available on the Nature Genetics website.

\section{ACKNOWLEDGMENTS}

We thank J. Feramisco for continuous support to the project and for providing the microinjection facility; M. Montminy for support to P.L.P. and C.S.; C. Buckmaster for technical assistance in microinjection; J. Feramisco, V. Sartorelli, J.Y.J. Wang and members of the laboratory of P.L.P. for discussions and critical reading of the manuscript; V. Sartorelli for antibodies to acetylated MyoD; E. Knudsen for BRM, BRG1 and BRG1 (K798R) enzymatic-deficient mutant cDNA; J. Han for antibodies to MEF2c; T. Sudo for antibodies to p38 $\alpha$; and R. Evans for SW13 cells. This project was partially supported by MDA (Research Developmental Grant to P.L.P.) and by Compagnia San Paolo di Torino to Dulbecco Telethon Institute. C.S. was supported by a Telethon fellowship and Sbarro Health Research Organization. S.V.F. is supported by a Marie Curie fellowship.

\section{COMPETING INTERESTS STATEMENT}

The authors declare that they have no competing financial interests.

Received 8 March; accepted 4 May 2004

Published online at http://www.nature.com/naturegenetics/

1. Sartorelli, V. \& Puri, P.L. The link between chromatin structure, protein acetylation and cellular differentiation. Front. Biosci. 6, D1024-D1047 (2001).

2. McKinsey, T.A., Zhang, C.L. \& Olson, E.N. Signaling chromatin to make muscle. Curr. Opin. Cell Biol. 14, 763-772 (2002).

3. Cuenda, A. \& Cohen, P. Stress-activated protein kinase-2-p38 and a rapamycin-sensitive pathway are required for C2C12 myogenesis. J. Biol. Chem. 274, 4341-4346 (1999).

4. Zetser, A., Gredinger, E. \& Bengal, E. p38 mitogen-activated protein kinase pathway promotes skeletal muscle differentiation. Participation of the Mef2c transcription factor. J. Biol. Chem. 274, 5193-5200 (1999).

5. Wu, Z. et al. p38 and extra-cellular signal-regulated kinases regulate the myogenic program at multiple steps. Mol. Cell. Biol. 20, 3951-3964 (2000).

6. Puri, P.L. et al. Induction of terminal differentiation by constitutive activation of $p 38$ MAP kinase in Human rhabdomyosarcomas. Genes Dev. 14, 574-584 (2000).

7. Raingeaud, J. et al. MKK3- and MKK6-regulated gene expression is mediated by the p38 mitogen activated protein kinase signal transduction pathway. Mol. Cell. Biol. 16, 1247-1255 (1996).

8. Bergstrom, D.A. et al. Promoter-specific regulation of MyoD binding and signal transduction cooperate to pattern gene expression. Mol. Cel/ 3, 587-600 (2002).

9. Han, J. et al. Activation of the transcription factor MEF2C by the MAP kinase p38 in inflammation. Nature 386, 296-299 (1997). 
10. Zhao, M. et al. Regulation of the MEF2 family of transcription factors by p38. Mol Cell. Biol. 19, 21-30 (1999).

11. Puri, P.L. \& Sartorelli, V. Regulation of muscle regulatory factors by DNA-binding, interacting proteins, and post-transcriptional modifications. J. Cell Physiol. 185, 155-173 (2000)

12. Puri, P.L. et al. Differential roles of $\mathrm{p} 300$ and PCAF acetyltransferases in muscle differentiation. Mol. Cell 1, 35-45 (1997b).

13. de la Serna, I.L., Carlson, K.A. \& Imbalzano, A.N. Mammalian SWI-SNF complexes promote MyoD-mediated muscle differentiation. Nat. Genet. 27, 187-190 (2001).

14. Saccani, S., Pantano, S. \& Natoli, G. p38-Dependent marking of inflammatory genes for increased NF-kappa B recruitment. Nat. Immunol. 1, 69-75 (2002).

15. Sartorelli, V. et al. Acetylation of MyoD directed by PCAF is necessary for the execu tion of the muscle program. Mol. Cell 4, 725-734 (1999).

16. Polesskaya, A., et al. CREB-binding Protein-p300 activates MyoD by acetylation. J. Biol. Chem. 275, 34359-34364 (2000).

17. Fulco, M. et al. Sir2 regulates skeletal muscle differentiation as a potential sensor of the redox state. Mol. Cell 12, 51-62 (2003).

18. Roy, K., de la Serna, I.L. \& Imbalzano, A.N. The myogenic basic helix-loop-helix family of transcription factors shows similar requirements for SWI-SNF chromatin remodeling enzymes during muscle differentiation in culture. J. Biol. Chem. 277, 33818-33824 (2002).

19. Gerber, A.N., Klesert, T.R., Bergstrom D.A. \& Tapscott, S.J. Two domains of MyoD mediate transcriptional activation of genes in repressive chromatin: a mechanism for lineage determination in myogenesis. Genes Dev. 11, 436-450 (1997).

20. Wilson, C.J. et al. RNA polymerase II holoenzyme contains SWI-SNF regulators involved in chromatin remodeling. Cel/ 2, 235-244 (1996).

21. Black, B.L., Molkentin, J.D. \& Olson, E. Multiples roles for the MyoD basic region in transmission of transcriptional activation signals and interaction with Mef2. Mol. Cell. Biol. 18, 69-77 (1998).

22. Hsiao, P.W., Fryer, C.J., Trotter, K.W., Wang, W. \& Archer, T.K. BAF60a mediates critical interactions between nuclear receptors and the BRG1 chromatin-remodeling complex for transactivation. Mol. Cell. Biol. 23, 6210-6220 (2003).

23. Debril, M.B. et al. Transcription factors and nuclear receptors interact with the SWISNF complex through the BAF60c subunit. J. Biol. Chem. 16, 16677-16686 (2003).

24. Muchardt, C. \& Yaniv, M. A human homologue of Saccharomyces cerevisiae SNF2 SWI2 and Drosophila brm genes potentiates transcriptional activation by the glucocorticoid receptor. EMBO J. 11, 4279-4290 (1993).

25. Khavari, P.A., Peterson, C.L., Tamkun, J.W., Mendel, D.B. \& Crabtree, G.R. BRG1 contains a conserved domain of the SWI2-SNF2 family necessary for normal mitotic growth and transcription. Nature 366, 170-174 (1993).

26. Neely, K.E. et al. Transcription activator interactions with multiple SWI-SNF subunits. Mol. Cell. Biol. 6, 1615-1625 (2002)

27. Kadam, S. \& Emerson, B.M. Transcriptional specificity of human SWI-SNF BRG1 and BRM chromatin remodeling complexes. Mol. Cell 2, 377-389 (2003).

28. Hassan, A.H., Neely, K.E. \& Workman, J. Histone acetyltransferases complexes stabilize SWI-SNF binding to promoter nucleosomes. Cell 104, 817-827 (2001).

29. Sif, S., Stukenberg, P.T., Kirschner, M.W. \& Kingston, R.E. Mitotic inactivation of a human SWI-SNF chromatin remodeling complex. Genes Dev. 18, 2842-2851 (1998). 